\title{
Perfusion-CT guided intravenous thrombolysis in patients with unknown-onset stroke: a randomized, double-blind, placebo-controlled, pilot feasibility trial
}

\author{
Patrik Michel • George Ntaios • Marc Reichhart • \\ Christian Schindler • Julien Bogousslavsky • \\ Philip Maeder • Reto Meuli • Max Wintermark
}

Received: 23 March 2011 /Accepted: 25 July 2011 /Published online: 2 August 2011

(C) Springer-Verlag 2011

\begin{abstract}
Introduction Patients with unknown stroke onset are generally excluded from acute recanalisation treatments. We designed a pilot study to assess feasibility of a trial of perfusion computed tomography (PCT)-guided thrombolysis in patients with ischemic tissue at risk of infarction and unknown stroke onset.

Methods Patients with a supratentorial stroke of unknown onset in the middle cerebral artery territory and significant volume of at-risk tissue on PCT were randomized to intravenous thrombolysis with alteplase $(0.9 \mathrm{mg} / \mathrm{kg})$ or placebo. Feasibility endpoints were randomization and blinded treatment of patients within $2 \mathrm{~h}$ after hospital arrival, and the correct application (estimation) of the perfusion imaging criteria.

Results At baseline, there was a trend towards older age [69.5 (57-78) vs. $49(44-78)$ years] in the thrombolysis group $(n=6)$ compared to placebo $(n=6)$. Regarding feasibility, hospital arrival to treatment delay was above
\end{abstract}

P. Michel $\cdot$ G. Ntaios $\cdot$ M. Reichhart

Department of Neurology Service, Center Hospitalier

Universitaire Vaudois and University of Lausanne,

Lausanne, Switzerland

\section{Schindler}

Pharmacy Department, Center Hospitalier Universitaire Vaudois and University of Lausanne,

Lausanne, Switzerland

\section{J. Bogousslavsky}

Genolier Swiss Medical Network,

Clinique Valmont,

Glion, Switzerland the allowed $2 \mathrm{~h}$ in three patients (25\%). There were two protocol violations $(17 \%)$ regarding PCT, both underestimating the predicted infarct in patients randomized in the placebo group. No symptomatic hemorrhage or death occurred during the first 7 days. Three of the four $(75 \%)$ and one of the five $(20 \%)$ patients were recanalized in the thrombolysis and placebo group respectively. The volume of non-infarcted at-risk tissue was $84(44-206) \mathrm{cm}^{3}$ in the treatment arm and $29(8-105) \mathrm{cm}^{3}$ in the placebo arm. Conclusions This pilot study shows that a randomized PCTguided thrombolysis trial in patients with stroke of unknown onset may be feasible if issues such as treatment delays and reliable identification of tissue at risk of infarction tissue are resolved. Safety and efficiency of such an approach need to be established.

Keywords Perfusion CT Thrombolysis $\cdot$ Stroke of unknown onset

P. Maeder $\cdot$ R. Meuli

Department of Radiology, Center Hospitalier Universitaire

Vaudois and University of Lausanne,

Lausanne, Switzerland

M. Wintermark

Department of Radiology, Division of Neuroradiology,

University of Virginia,

Charlottesville, VA, USA

P. Michel $(\bowtie)$

Neurology Service, Centre Hospitalier Universitaire Vaudois

\& University of Lausanne,

Rue du Bugnon 46,

1011 Lausanne, Switzerland

e-mail: patrik.michel@chuv.ch 


\section{Introduction}

The implementation of intravenous thrombolysis with recombinant tissue plasminogen activator (rTPA) in the late 1990s after the results of the National Institute of Neurological Disorders and Stroke rtPA Stroke trial [1] was a cornerstone in the acute management of ischemic stroke. The results of the European Cooperative Acute Stroke Study (ECASS) III [2] and the Prolyse in Acute Cerebral Thromboembolism II trial (PROACT II) [3] may allow for a longer time window for acute recanalisation if approved by authorities, although the publication of these studies has already lead to a rapid and lasting increase of thrombolysis in acute stroke patients [4]. The reasons why a majority of patients do not receive thrombolytic treatment are late hospital arrival and unknown time of stroke onset. For example, $33.1 \%$ of all stroke patients admitted to our institution between 2003 and 2008 were of unknown onset, with the typical case being wake-up strokes or aphasic patients who suffered a stroke without any witness [5]. The only trial specifically aimed at treating wake-up stroke patients has, based on standard neuroimaging, produced negative results [6].

Modern imaging like perfusion computed tomography (PCT) and diffusion and perfusion-weighted magnetic resonance (DWI-PWI-MRI) have been investigated for prediction of tissue infarction. On MRI for example, final infarct can be predicted with rather good accuracy with acute DWI [7], and with very high accuracy with subacute fluidattenuated inversion recovery [8]. Properly executed PCT is well tolerated, readily available, and is currently performed in many centers as part of a standard CT protocol, which also includes non-contrast $\mathrm{CT}$ and $\mathrm{CT}$ angiography [9]. Although it requires further validation [10], systematic evaluations of thresholds have been performed to predict tissue infarction and identify at-risk ischemic brain $[11,12]$.

Up to now, all mismatch-based thrombolysis trials set an upper threshold for the onset-to-intervention interval, which ranged from $6 \mathrm{~h}[13,14]$ to $9 \mathrm{~h}[15-17]$. On the contrary, no randomized trial has yet been performed in unknown-onset stroke patients, although a at-risk tissue may still persist up to $18 \mathrm{~h}$ in $44.1 \%$ of patients [18] and in $37.1 \%$ of wake-up patients [19].

The main aim of this pilot study was to assess the feasibility of performing a randomized PCT-guided thrombolysis trial in patients with a limited volume of predicted tissue infarct, significant volumes of tissue at risk of infarction, and unknown stroke onset.

\section{Methods}

This was a single-center, prospective, randomized, doubleblinded, placebo-controlled, phase II study to investigate the feasibility of r-TPA administration in patients with acute ischemic stroke of unknown onset and a large volume of atrisk brain tissue assessed by PCT. The study was approved by the hospital ethics committee. Signed informed consent was obtained from all patients or a proxy if the patient could not be consented. This study was not registered in $\mathrm{http}: / / w w w . c l i n i c a l t r i a l s . g o v$ because it was initiated before 2005 (when the registration of randomized controlled trials became mandatory). This report was written in accordance with the CONSORT reporting guidelines [20, 21].

\section{Study population}

Patients with a supratentorial stroke including the territory of the middle cerebral artery were eligible for the study. Stroke onset was considered "unknown" when the exact time of stroke onset was estimated with more than $1 \mathrm{~h}$ uncertainty. Furthermore, hospital arrival had to be too late for standard IV thrombolysis within $3 \mathrm{~h}$ since last proof of good health, which was the time limit recommended by professional societies and accepted by national authorities during the study period. Finally, last proof of well-being had to be not more than $24 \mathrm{~h}$. The other inclusion and exclusion criteria are presented in Table 1. Based on expected low chance of finding patient fulfilling all inclusion/exclusion criterion in a single center, and the pilot character of the study, we aimed at recruiting 12 patients over a 2-year period. All clinical outcomes were assessed by National Institute of Health Stroke Scale score (NIHSS)- and Rankin-certified stroke neurologists.

\section{Imaging protocol}

CT, PCT, and CTA were performed on a General Electrics Lightspeed 16 advantage (16 detectors; General Electric Company, Fairfield, CT, USA) until November 2005, and a General Electrics Lightspeed VCT (64 detectors) thereafter, using their commercially available software. For the PCT, acquisition delay was $7 \mathrm{~s}$ after injection of $50 \mathrm{ml}$ of iohexol (300 mg/ml of iodine; Accupaque 300, Amersham, Oslo, Norway) with a rate of injection of $5 \mathrm{ml}$ per second into an antecubital vein using a power injector $\left(\right.$ Stellant ${ }^{\circledR}$ DCT Injection System; Medrad, Warrendale, PA, USA). Two injections with acquisition of two $10-\mathrm{mm}$ slices each were acquired. The PCT data were transferred to a workstation and analyzed using perfusion analysis software to create parametric maps of mean transit time (MTT), cerebral blood flow (CBF), and cerebral blood volume (CBV) map utilizing a deconvolution software developed by Philips Medical Systems (Cleveland, OH, USA). The CTPpredicted infarct and at-risk ischemic tissue were semiautomatically calculated by the software using the appropriate MTT and CBV thresholds (MTT $>145 \%$ of the 
Table 1 Inclusion and exclusion criteria

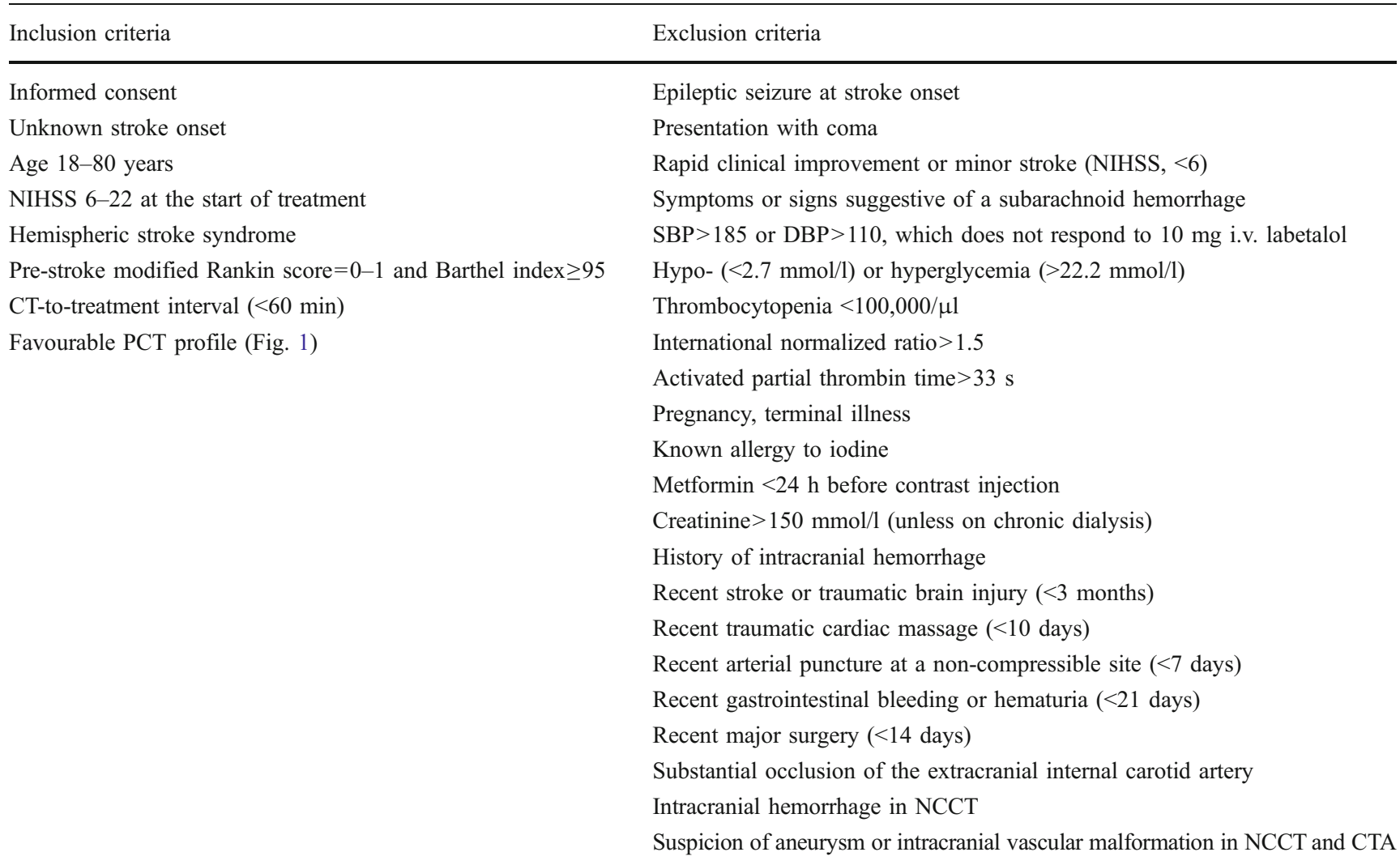

NIHSS National Institute of Health Stroke Scale score, CT computed tomography, NCCT non-contrast CT, CTA CT angiography, SBP/DBP systolic/diastolic blood pressure, $P C T$ perfusion CT

contralateral side values and $\mathrm{CBV}<2.0 \mathrm{~mL} / 100 \mathrm{~g}$ to define PCT-predicted infarct core; MTT $>145 \%$ of the contralateral side values and $\mathrm{CBV}>2.0 \mathrm{~mL} / 100 \mathrm{~g}$ to define at-risk ischemic tissue) [11]. These thresholds, although expressed partially as absolute values, are relative values and depend on the acquisition and calculation software. These reconstructions require the radiologist to assess the quality of input and output function and to select cerebral arteries and veins for the deconvolution process

In the acute situation, reconstruction of PCT was performed by the on-call radiologist in training, and a visual estimation of predicted infarct and at-risk ischemic tissue was performed after semi-automated reconstruction by a stroke neurologist (PM) and the same radiologist. For the final analysis, images were reconstructed by an experienced neuroradiologist (MW). In addition to measuring the volume of the predicted infarct and of the tissue at risk using the computer software, he compared the ischemic volumes (predicted infarct and tissue at risk) with the entire MCA territory of the individual patient and expressed this as percentages. For this, $100 \%$ of the MCA territory was determined for each patient using estimated borders of the territory.
CTA was performed immediately before or after CTP; results of CTA were not used as an inclusion/exclusion criterion.

\section{Selection criteria based on perfusion CT}

We used systemically evaluated thresholds to generate PCT images, which allow approximate prediction of infarct and of tissue at risk [11]. It has been shown that the benefit of thrombolysis (a) is reciprocally associated with the extent of the initially predicted infarct volume and becomes unfavorable when this exceeds $30-40 \%$ of the territory of the middle cerebral artery (MCA) $[22,23]$ and (b) increases with a high ratio of tissue at risk/predicted infarct $[13,14]$. Based on these findings, we set an upper limit for the extent of predicted infarction, which should not exceed $30 \%$ of the MCA territory. In addition, we set a variable threshold for the extent of the at-risk brain tissue as shown in Fig. 1, where stroke index is defined as at-risk tissue/( at-risk tissue + predicted infarct): With little predicted infarct and relatively small at-risk tissue, thrombolysis was considered worth-performing and safe, whereas in case of a larger predicted infarct, the patient should have more at-risk tissue 
to be treated. This concept was chosen to limit the risk of bleeding and increase the chances of a treatment response.

\section{Treatment protocol}

The rTPA (Actilyse ${ }^{\circledR}$ Boehringer Ingelheim GmbH, Germany) was administered intravenously at a dose of $0.9 \mathrm{mg} /$ $\mathrm{kg}$ of body weight (maximum of $90 \mathrm{mg}$ ), with $10 \%$ of the dose as a bolus infusion and the rest $90 \%$ as an hourly infusion. Placebo consisted of lyophilized powder produced in the hospital's pharmacy. Both regimens (rTPA and placebo) were placed in identical bottles by the hospital pharmacy and were indistinguishable in appearance. The general management of patients followed international guidelines valid during the study period [24].

Randomization and allocation to treatment

Randomization was performed on a 1:1 basis according to a random number table generated by an independent hospital pharmacist who was not involved in patient management and outcome assessment. Enrollment of patients and allocation to treatment was performed by blinded treating physicians. All medical personnel involved in the study were blinded during treatment and outcome assessment, except for the pharmacy. Unblinding was performed by the hospital pharmacy after all patients had completed their follow-up of 3 months.

\section{Primary outcome}

The main aim of the study was feasibility, as measured by (a) delay from hospital arrival to treatment $\leq 2 \mathrm{~h}$ and (b) the correct application of the PCT-inclusion criteria (details see below) in all patients. The rather long arrival-to-treatment delay was chosen because it included acquisition and interpretation of perfusion imaging, informed consent, and the randomization process.

\section{Secondary outcomes}

Secondary endpoints included symptomatic and asymptomatic intracerebral hemorrhage at $24 \mathrm{~h}$ and 4 days after treatment (assessed by CT or MRI respectively) according to the ECASS II criteria [22] and mortality at 7 and 90 days.

We also assessed the volume of at-risk brain tissue on PCT, which survived as compared to the T2W MRI lesion at day 4 , complete recanalisation (defined as no or $<50 \%$ narrowing on the control CTA at $24 \mathrm{~h}$ in all initially occluded arteries), neurological outcome (NIHSS) at 1, 7, and 90 days, and dependency (modified Rankin score and Barthel index) at 90 days.

\section{Monitoring}

Patients were monitored closely in the intermediate care stroke unit for clinical worsening and complications and appropriate examinations were performed if this occurred. At $24 \mathrm{~h}$, repeat non-contrast CT with circle of Willis CT angiography was performed in all patients, and at 4 days, cerebral MRI with MR angiography was obtained in all patients. An interruption of the study was pre-planned if more than three patients suffered symptomatic hemorrhage. In such case, the pharmacy department would have broken
Fig. 1 Patients were eligible for inclusion in the study if the values of predicted infarct size and stroke index were in the green area. On the contrary, values at the red area lead to exclusion from the study because thrombolysis in these situations was considered to be potentially unsafe. Stroke index: at-risk tissue/(at-risk tissue+ predicted infarct). $M C A$ Middle cerebral artery. Asterisk and plus symbol correspond to patients randomized to placebo and rTPA respectively

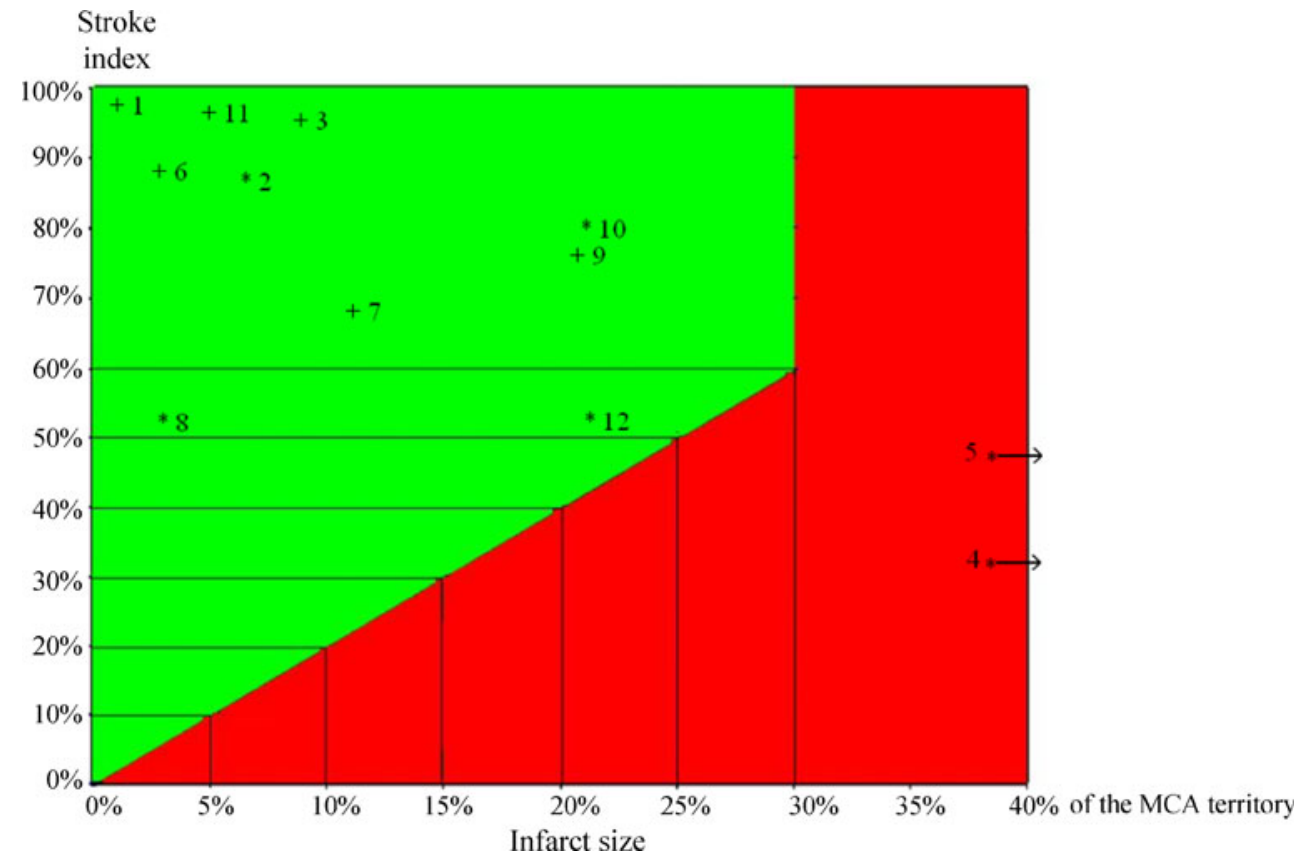




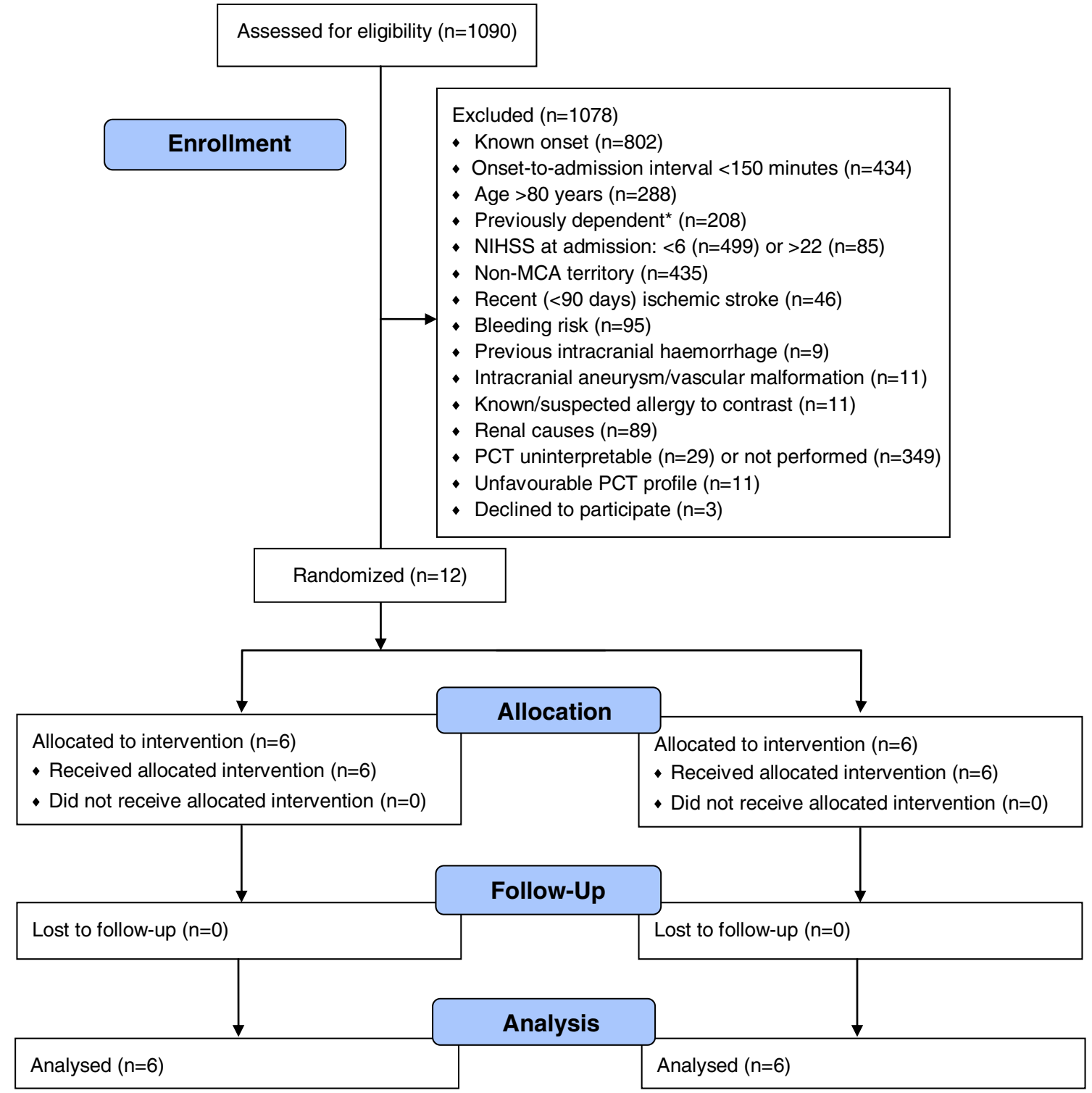

Fig. 2 Study flow diagram. Patients may have had multiple reasons for not being eligible for the trial. NIHSS National Institute of Health Stroke Scale score, $M C A$ middle cerebral artery, $L M W H$ low molecular

the randomization code of the already enrolled patients and informed two board-certified neurologists not participating in the study so that they could evaluate whether symptomatic hemorrhage (or death) was attributable to rtPA and whether the study should be terminated.

\section{Statistical analysis}

Analysis was performed on an intention-to-treat basis. Continuous variables are reported as median (range). Comparisons were performed by Fisher's exact test for categorical variables and Wilcoxon test for continuous variables. The level of significance was set at $95 \%(p=0.05)$. The statistical weight heparin, INR international normalized ratio, $P C T$ perfusion $\mathrm{CT}$, $M R I$ magnetic resonance imaging, $r T P A$ recombinant tissue plasminogen activator. Asterisk Previous modified Rankin score $\leq 2$

analysis was performed with Stata Statistical Software (Release 10. College Station, TX, USA: StataCorp LP 2007).

\section{Results}

Of the 1090 consecutive patients with an acute $(<24 \mathrm{~h}$ after symptom onset) ischemic stroke admitted to the stroke unit or intensive care of our institution between 06/2004 and 12/ 2007 (3.5 years), 12 patients were randomized to rTPA or placebo (Fig. 2). Of the randomized patients, nine (75\%) suffered a wake-up stroke (four in the rTPA group and five in the placebo group). The others were strokes with 
unknown onset with last proof of well-being during daytime. The ischemic lesion affected the right hemisphere in four patients in the placebo group and two in the active treatment arm. There were no significant differences in gender, NIHSS at admission, and time interval between last proof of well-being and onset of treatment between the active treatment and the placebo groups. However, there was a trend towards older age and higher NIHSS in the active treatment arm and to higher predicted infarct volumes in the placebo group (Table 2).

Door-to-needle time was a median of $113(75-205) \mathrm{min}$, and three patients received treatment or placebo beyond the goal of $2 \mathrm{~h}$ : two patients in the placebo group (at 138 and $205 \mathrm{~min}$.) and one in the rtPA group (131 min).

Reanalysis of PCT data after the end of the study showed protocol violations in two patients, both in the placebo arm. They were randomized based on favorable appearing perfusion profiles in the emergency room. The reanalyzed predicted infarct volumes of these two patients in fact exceeded the threshold described in Fig. 1 and were beyond $30 \%$ of the MCA territory

No patients were lost to follow-up. Regarding safety, there was no symptomatic intracranial hemorrhage or death during the first 7 days after randomization, and there were five asymptomatic hemorrhagic transformations at 4 days (three in the rTPA group and two in the placebo group). The volume of noninfarcted at-risk tissue and recanalization rates were higher in the active treatment arm. Neurological and clinical outcome in patients treated with rTPA was not worse compared to placebo patients (Table 3). Figure 3 presents an example of a patient who was randomized to, and has received rt-PA, with subsequent recanalisation and favorable outcome.

\section{Discussion}

This pilot study shows that a randomized PCT-guided thrombolysis trial in patients with stroke of unknown onset is partially feasible. Difficulties included protocol violations regarding door-to-needle time in $25 \%$ of patients and in respecting the prespecified perfusion-imaging criteria in $16.7 \%$ of patients.

Adding perfusion and arterial imaging criteria to decisions on treatment strategy is likely to increase the delay to treatment initiation. Given the importance of time in traditional thrombolysis protocols [25], such additional imaging needs to be shown feasible and of additive value $[26,27]$. Our median door-to-needle time of 113 (75-205) min may be partially explained by the need for reconstruction of PCT threshold maps, signed informed consent, and randomization procedures. It may show limits of the feasibility of such a thrombolysis protocol and should clearly be reduced in future trials. Secondly, the fact that initial calculations of PCT volumes performed at the emergency room were imprecise in two out of 12 patients (and hence, lead to an incorrect inclusion) is of concern and needs to be improved. Although this rate is similar to the rate of protocol violations of the non-contrast $\mathrm{CT}$ based " $1 / 3 \mathrm{MCA}$ territory rule" observed in another, large thrombolysis trial [23], the recent CT-based ECASS-3 trial had a low rate of radiological protocol violations of only $2.1 \%$ [2]. Thirdly, the recruitment period had to be enlarged from the planned 2-3.5 years to recruit the 12 patients in a single center, hinting at a rather restricted number of eligible and patients for such a trial.

A recent combined analysis of randomized thrombolysis trials found no convincing evidence to support that the risk of serious or fatal symptomatic hemorrhage increases with late initiation of thrombolysis [25]. In this sense, the main argument against late administration of rTPA in stroke patients would be the potential lack of efficacy, which could be due to lack of at-risk tissue or more resistance to recanalizing occluded arteries. Hypothetically, the demonstration of at-risk tissue may be as important as time in order to identify responders to acute recanalisation treatments. This concept seems to be supported by data from patients treated within $6 \mathrm{~h}$ who underwent perfusion imaging before thrombolysis $[13,14]$.

We also restricted our study to patients with small volumes of predicted infarct, which may allow limiting the hemorrhage risk [28]. Indeed, no symptomatic hemorrhage or death occurred in our study during the first 7 days after rTPA administration, but our number of patients treated is too small to assume safety with this protocol. When assessing recanalisation, growth from predicted to final infarct and survival of at-risk tissue as a potential surrogate marker for efficacy of thrombolysis, we found no signs of adverse effects.

The only randomized controlled perfusion-imagingbased thrombolysis study so far did not show a benefit in a late-time window [17]. The employment in the present study of a variable threshold incorporating not only the relative size of at-risk tissue but also the initial infarct size prediction may have lead to a more optimal selection of patients. Although a combination of non-contrast CT, CTA source imaging, and assessment of collaterals may be an alternative strategy for identifying patients with particular benefits of risk of (late) recanalisation, the advantage of using semi-automated threshold-based CTP reconstructions is that quantification of infarct prediction and at-risk tissue becomes less operator dependent because there it is (a) less subject to subjective visual estimations and (b) has predefined thresholds, which have been identified by systematic analysis of CTP generated values [11, 12]. On the other hand, using sophisticated perfusion imaging criteria for patient selection may severely limits the pool of patients that are eligible for treatment. 
Table 2 Baseline characteristics

Demographics, time intervals

Age (years)

Female gender

Prehospital modified Rankin score $=0$

LPW-to-treatment time (minutes)

Admission-to-treatment time (minutes)

Wake-up strokes

Vital signs

Temperature $\left({ }^{\circ} \mathrm{C}\right)$

Systolic blood pressure $(\mathrm{mmHg})$

Diastolic blood pressure $(\mathrm{mmHg})$

Heart rate $\left(\mathrm{min}^{-1}\right)$

Laboratory values

Serum creatinine $(\mu \mathrm{mol} / \mathrm{l})$

Glucose (mmol/l)

Total cholesterol ( $\mathrm{mmol} / \mathrm{l})$

White blood cell count $\left(10^{3} / \mathrm{mm}^{3}\right)$

Neuroimaging studies

Initially predicted infarct $\left(\mathrm{cm}^{3}\right)$

Initial at-risk tissue

Arterial imaging on admission

MCA segment 1 or 2 occlusion

Extracranial carotid occlusion

Clinical examination

NIHSS at admission

NIHSS at $4-6 \mathrm{~h}$

NIHSS at $24 \mathrm{~h}$

Risk factors and medical history

Hypertension

Atrial fibrillation

Dyslipidemia

Diabetes mellitus

Mechanical or biological valve

Coronary artery

Active smoking

Periphery artery disease

Previous treatment

Antiplatelet treatment

Anticoagulant

Antihypertensives

Oral hypoglycemic agents

Insulin

Lipid-lowering drugs

Stroke subtypes

Atherosclerotic

Cardioembolic

Dissection

Undetermined
$69.5(57-78)$

$3(50.0 \%)$

$6(100 \%)$

$564(390-805)$

$109.5(85-131)$

$4(66.7 \%)$

$36.0(34.2-37.0)$

153 (109-200)

83 (70-98)

$67(62-109)$

89 (66-104)

$6.4(5.7-16.8)$

$4.6(3.8-7.4)$

$9.5(5.4-12.7)$

$12.0(1.1-26.2)$

90.3 (30.5-207.8)

$5(50 \%)$

$4(67 \%)$

17 (13-21)

$13(8-19)$

$10.5(7-19)$

$4(66.7 \%)$

$2(33.3 \%)$

$4(66.7 \%)$

$1(16.7 \%)$

$-$

$-$

$-$

$-$

$2(33.3 \%)$

$-$

$3(50.0 \%)$

$-$

$-$

$2(33.3 \%)$

$2(33.3 \%)$

$2(33.3 \%)$

-

$2(33.3 \%)$
49 (44-78)

$3(50.0 \%)$

$5(83.3 \%)$

437.5 (330-656)

113 (75-205)

$5(83.3 \%)$

35.4 (34.4-36.1)

134 (100-196)

81.5 (65-92)

67 (50-92)

81 (68-99)

$7.0(5.5-9.6)$

$6.2(4.5-6.8)$

$10.0(4.2-13.1)$

$42.0(11.4-179.4)$

75.8 (60.4-114.6)

$4(67 \%)$

$1(17 \%)$

14.5 (12-19)

18 (11-23)

$19.5(6-24)$

$1(16.7 \%)$

$3(50.0 \%)$

$-$

$2(33.3 \%)$

$-$

$1(16.7 \%)$

$-$

$1(16.7 \%)$

$1(16.7 \%)$

$-$

$-$

$4(66.7 \%)$

$2(33.3 \%)$ 
Table 3 Rates of hemorrhagic transformation and mortality and indices of radiological and clinical outcome

\begin{tabular}{lll}
\hline Outcome & rTPA group $(n=6)$ & Placebo group $(n=6)$ \\
\hline Symptomatic hemorrhagic transformation at $24 \mathrm{~h} / 4$ days & $0 / 0$ & $0 / 0$ \\
Asymptomatic hemorrhagic transformations at $24 \mathrm{~h}(\mathrm{CT})$ & 2 & 2 \\
Asymptomatic hemorrhagic transformations at 4 days (MRI) & 3 & 2 \\
Mortality at $7 / 90$ days & $0 / 0$ & $0 / 0$ \\
Recanalization & $3 / 4^{\mathrm{c}}(75 \%)$ & $1 / 5^{\mathrm{c}}\left(20^{\circ}\right)$ \\
Non-infarcted at-risk tissue $\left(\mathrm{cm}^{3}\right)^{\mathrm{a}}$ & $84(44-206)$ & $29(8-105)$ \\
NIHSS & $4(0-6)$ & $9.5(6-15)$ \\
Barthel index & & $70(80-100)$ \\
Modified Rankin score & & $3.0(2-4)$ \\
Favorable functional outcome (Modified Rankin score $\leq 2)^{\mathrm{b}}$ & $95(30-95)$ & $1 / 6(16.6 \%)$ \\
\hline
\end{tabular}

For recanalization, non-infarted at-risk tissue, NIHSS Barthel index, and modified Rankin score: numbers represent median values (range)

rTPA Recombinant tissue plasminogen activator, NIHSS National Institute of Health Stroke Scale score

${ }^{a}$ Defined as the volume of at-risk tissue on PCT surviving as compared to the T2W MRI lesion at day 4

${ }^{\mathrm{b}}$ Assessed at 3 months

${ }^{\mathrm{c}}$ The denominator refers to patients with arterial occlusion diagnosed at admission

The results of the present study are limited by its small sample size, leading to a major inequalities in baseline variables, limited amount of information regarding feasi- bility, and insufficient power to study safety, biological efficacy (such as recanalisation or growth of predicted to final infarct), and clinical efficiency. Higher age, higher
Fig. 3 Example from a 75-yearold man with a wake-up stroke with aphasia and right hemisyndrome, NIHSS $=21$. Admission perfusion CT (a) performed $8 \mathrm{~h}$ $30 \mathrm{~min}$ after going to bed. Predicted infarct of $14.1 \mathrm{~cm}^{3}$ (red) and at-risk tissue of $207.8 \mathrm{~cm}^{3}$ (green) in the territory of the left middle cerebral artery due to an occlusion of the left MCA main stem (CTA, b). Randomized to intravenous thrombolysis, study drug started 9 h 5 min after last proof of well-being. Complete recanalisation was diagnosed at $24 \mathrm{~h}$ CTA (d) with a final infarct volume of $15.7 \mathrm{~cm}^{3}$ (5-day DWIMRI, c). At 3 months, modified Rankin score of 1 and Barthel index of 100

NIHSS, and lower predicted infarct in the rtPA group have potentially opposing influences on radiological and clinical outcomes, but again, numbers are too small for any meaningful adjustments or conclusions regarding efficacy. Secondly, systematic evaluation of infarct prediction by CTP has yielded different predictive threshold models based either on a combination of relative MTT and CBV [11] or on a relative CBF threshold [12]. Finally, arterial imaging was performed but not used for the selection process. This was done because (a) the presence of at-risk tissue on CTP despite the absence of large arterial occlusion may indicate thrombolysable distal vessel occlusion and (b) perfusion imaging is commonly regarded as the technically more challenging part in multimodal acute stroke imaging if compared with acute arterial imaging. Retrospective analysis of the DIAS-2 trial suggests that acute arterial occlusion may better identify thrombolysis responders than mismatch on perfusion imaging [17].

In summary, this pilot study shows that a PCT-guided randomized thrombolysis trial in patients with stroke of unknown onset may be feasible if issues such as delay from hospital arrival to treatment can be resolved and if early infarct prediction by perfusion imaging can be obtained reliably and accurately. Before starting a randomized trial with clinical efficiency as a primary outcome, additional safety and biological efficacy data should be obtained. Such a clinical efficiency trial would then require 357 patients to detect an absolute difference of $15 \%$ in favorable outcome (modified Rankin score, $\leq 2$ ) with a power of $80 \%$ and a two-sided $\alpha$ error of 5\%, assuming a rate of $55 \%$ favorable outcome in the thrombolysis arm [29] and a dropout rate of 5\%.

Acknowledgments A Swiss Cardiology Foundation grant funded parts of the salaries of research personnel involved in the study.

Conflict of interest PM receives funding from Paion and Lundbeck (research support and consulting fees) and Boehringer-Ingelheim (speaker fees, Advisory Board). MW receives funding from the National Center for Research Resources, Grant KL2 RR024130, GE Healthcare and Philips Medical Systems.

\section{References}

1. NINDS Study Group (1995) Tissue plasminogen activator for acute ischemic stroke. The National Institute of Neurological Disorders and Stroke rt-PA Stroke Study Group. New Engl J Med 333(24):1581-1587

2. Hacke W, Kaste M, Bluhmki E, Brozman M, Davalos A, Guidetti D et al (2008) Thrombolysis with alteplase 3 to 4.5 hours after acute ischemic stroke. N Engl J Med 359(13):1317-1329

3. Furlan A, Higashida R, Wechsler L, Gent M, Rowley H, Kase C et al (1999) Intra-arterial prourokinase for acute ischemic stroke. The PROACT II study: a randomized controlled trial. Prolyse in Acute Cerebral Thromboembolism. JAMA 282(21):2003-2011
4. Ahmed N, Wahlgren N, Grond M, Hennerici M, Lees KR, Mikulik R et al (2010) Implementation and outcome of thrombolysis with alteplase 3-4.5 h after an acute stroke: an updated analysis from SITS-ISTR. Lancet Neurol 9(9):866-874

5. Michel P, Odier C, Rutgers M, Reichhart M, Maeder P, Meuli R et al (2010) The Acute STroke Registry and Analysis of Lausanne (ASTRAL): design and baseline analysis of an ischemic stroke registry including acute multimodal imaging. Stroke 41(11):24912498

6. Adams HP Jr, Leira EC, Torner JC, Barnathan E, Padgett L, Effron $\mathrm{MB}$ et al (2008) Treating patients with 'wake-up' stroke: the experience of the AbESTT-II trial. Stroke 39(12):3277-3282

7. Chemmanam $\mathrm{T}$, Campbell $\mathrm{BC}$, Christensen $\mathrm{S}$, Nagakane $\mathrm{Y}$, Desmond PM, Bladin CF et al (2010) Ischemic diffusion lesion reversal is uncommon and rarely alters perfusion-diffusion mismatch 1. Neurology 75(12):1040-1047

8. Tourdias T, Renou P, Sibon I, Asselineau J, Bracoud L, Dumoulin $\mathrm{M}$ et al (2011) Final cerebral infarct volume is predictable by MR imaging at 1 week 1. AJNR Am J Neuroradiol 32(2):352-358

9. Wintermark M, Reichhart M, Cuisenaire O, Maeder P, Thiran JP, Schnyder P et al (2002) Comparison of admission perfusion computed tomography and qualitative diffusion- and perfusionweighted magnetic resonance imaging in acute stroke patients. Stroke 33(8):2025-2031

10. Wintermark M, Albers GW, Alexandrov AV, Alger JR, Bammer R, Baron JC et al (2008) Acute stroke imaging research roadmap. Stroke 39(5):1621-1628

11. Wintermark M, Flanders AE, Velthuis B, Meuli R, van Leeuwen M, Goldsher D et al (2006) Perfusion-CT assessment of infarct core and penumbra: receiver operating characteristic curve analysis in 130 patients suspected of acute hemispheric stroke. Stroke 37(4):979-985

12. Bivard A, McElduff P, Spratt N, Levi C, Parsons M (2011) Defining the extent of irreversible. Cerebrovascular Diseases 31 (3):238-245

13. Albers GW, Thijs VN, Wechsler L, Kemp S, Schlaug G, Skalabrin $\mathrm{E}$ et al (2006) Magnetic resonance imaging profiles predict clinical response to early reperfusion: the diffusion and perfusion imaging evaluation for understanding stroke evolution (DEFUSE) study. Ann Neurol 60(5):508-517

14. Davis SM, Donnan GA, Parsons MW, Levi C, Butcher KS, Peeters A et al (2008) Effects of alteplase beyond $3 \mathrm{~h}$ after stroke in the Echoplanar Imaging Thrombolytic Evaluation Trial (EPITHET): a placebo-controlled randomised trial. Lancet Neurol 7 (4):299-309

15. Furlan AJ, Eyding D, Albers GW, Al-Rawi Y, Lees KR, Rowley HA et al (2006) Dose Escalation of Desmoteplase for Acute Ischemic Stroke (DEDAS): evidence of safety and efficacy 3 to 9 hours after stroke onset. Stroke 37(5):1227-1231

16. Hacke W, Albers G, Al-Rawi Y, Bogousslavsky J, Davalos A, Eliasziw $M$ et al (2005) The Desmoteplase in Acute Ischemic Stroke Trial (DIAS): a phase II MRI-based 9-hour window acute stroke thrombolysis trial with intravenous desmoteplase. Stroke 36 (1):66-73

17. Hacke W, Furlan AJ, Al-Rawi Y, Davalos A, Fiebach JB, Gruber F et al (2009) Intravenous desmoteplase in patients with acute ischaemic stroke selected by MRI perfusion-diffusion weighted imaging or perfusion CT (DIAS-2): a prospective, randomised, double-blind, placebo-controlled study. Lancet Neurol 8(2):141-150

18. Darby DG, Barber PA, Gerraty RP, Desmond PM, Yang Q, Parsons M et al (1999) Pathophysiological topography of acute ischemia by combined diffusion-weighted and perfusion MRI. Stroke 30 (10):2043-2052

19. Silva GS, Lima FO, Camargo EC, Smith WS, Singhal AB, Greer $\mathrm{DM}$ et al (2010) Wake-up stroke: clinical and neuroimaging characteristics. Cerebrovasc Dis 29(4):336-342 
20. Schulz KF, Altman DG, Moher D (2010) CONSORT 2010 statement: updated guidelines for reporting parallel group randomized trials. Ann Intern Med 152(11):726-732

21. Moher D, Hopewell S, Schulz KF, Montori V, Gotzsche PC, Devereaux PJ et al (2010) CONSORT 2010 Explanation and Elaboration: Updated guidelines for reporting parallel group randomised trials. J Clin Epidemiol 63(8):e1-e37

22. Hacke W, Kaste M, Fieschi C, von Kummer R, Davalos A, Meier $\mathrm{D}$ et al (1998) Randomised double-blind placebo-controlled trial of thrombolytic therapy with intravenous alteplase in acute ischaemic stroke (ECASS II). Second European-Australasian Acute Stroke Study Investigators. Lancet 352(9136):1245-1251

23. Hacke W, Kaste M, Fieschi C, Toni D, Lesaffre E, von Kummer R et al (1995) Intravenous thrombolysis with recombinant tissue plasminogen activator for acute hemispheric stroke. The European Cooperative Acute Stroke Study (ECASS). JAMA 274(13):10171025

24. Toni D, Chamorro A, Kaste M, Lees K, Wahlgren NG, Hacke W (2004) Acute treatment of ischaemic stroke. European Stroke Initiative (EUSI). Cerebrovasc Dis 17(Suppl 2):30-46
25. Lees KR, Bluhmki E, von Kummer R, Brott TG, Toni D, Grotta JC et al (2010) Time to treatment with intravenous alteplase and outcome in stroke: an updated pooled analysis of ECASS, ATLANTIS, NINDS, and EPITHET trials. Lancet 375 (9727):1695-1703

26. Mishra NK, Albers GW, Davis SM, Donnan GA, Furlan AJ, Hacke W et al (2010) Mismatch-based delayed thrombolysis: a meta-analysis. Stroke 41(1):e25-e33

27. Obach V, Oleaga L, Urra X, Macho J, Amaro S, Capurro S et al (2011) Multimodal CT-assisted thrombolysis in patients with acute stroke. A cohort study. Stroke 42:1129-1131

28. Tong DC, Adami A, Moseley ME, Marks MP (2000) Relationship between apparent diffusion coefficient and subsequent hemorrhagic transformation following acute ischemic stroke. Stroke 31 (10):2378-2384

29. Wahlgren N, Ahmed N, Davalos A, Ford GA, Grond M, Hacke W et al (2007) Thrombolysis with alteplase for acute ischaemic stroke in the Safe Implementation of Thrombolysis in StrokeMonitoring Study (SITS-MOST): an observational study. Lancet 369(9558):275-282 